Poincare Journal of Analysis $\&$ Applications

Vol. 2018 (1), 1-8

(C) Poincare Publishers

OINCAR

UBLISH $2 \overline{\overline{R S}}$

\title{
Properties of almost $\mathcal{I}$-continuous functions
}

\author{
S. JAFARI ${ }^{\dagger}$, R. SARANYA AND N. RAJESH
}

$\begin{array}{lll}\text { Date of Receiving } & : & 13.08 .2017 \\ \text { Date of Revision } & : & 02.10 .2017 \\ \text { Date of Acceptance } & : & 07.03 .2018\end{array}$

\begin{abstract}
The aim of this paper is to introduce and characterize a new class of functions called almost $\mathcal{I}$-continuous functions in ideal topological spaces by using $\mathcal{I}$-open sets.
\end{abstract}

\section{Introduction}

The concept of ideals in topological spaces has been introduced and studied by Kuratowski [11] and Vaidyanathaswamy, [19]. An ideal $\mathcal{I}$ on a topological space $(X, \tau)$ is a nonempty collection of subsets of $X$ which satisfies (i) $A \in \mathcal{I}$ and $B \subset A$ implies $B \in \mathcal{I}$ and (ii) $A \in \mathcal{I}$ and $B \in \mathcal{I}$ implies $A \cup B \in \mathcal{I}$. Given a topological space $(X, \tau)$ with an ideal $\mathcal{I}$ on $X$ and if $\mathcal{P}(X)$ is the set of all subsets of $X$, a set operator $(.)^{\star}$ : $\mathcal{P}(X) \rightarrow \mathcal{P}(X)$, called the local function [19] of $A$ with respect to $\tau$ and $\mathcal{I}$, is defined as follows: for $A \subset X, A^{\star}(\tau, \mathcal{I})=\{x \in X \mid U \cap A \notin \mathcal{I}$ for every $U \in \tau(x)\}$, where $\tau(x)=$ $\{U \in \tau: x \in U\}$. A Kuratowski closure operator $\mathrm{Cl}^{\star}(\cdot)$ for a topology $\tau^{\star}(\tau, \mathcal{I})$ called the $\star$-topology, finer than $\tau$ is defined by $\mathrm{Cl}^{\star}(A)=A \cup A^{\star}(\tau, \mathcal{I})$ when there is no chance of confusion, $A^{\star}(\mathcal{I})$ is denoted by $A^{\star}$. If $\mathcal{I}$ is an ideal on $X$, then $(X, \tau, \mathcal{I})$ is called an ideal topological space. The aim of this paper is to introduce and characterize a new class of functions called almost $\mathcal{I}$-continuous functions in ideal topological spaces by using $\mathcal{I}$-open sets.

\section{Preliminaries}

Let $A$ be a subset of a topological space $(X, \tau)$. We denote the closure of $A$ and the interior of $A$ by $\mathrm{Cl}(A)$ and $\operatorname{Int}(A)$, respectively. A subset $A$ of a topological space $(X, \tau)$ is said to be regular open [18] if $A=\operatorname{Int}(\mathrm{Cl}(A))$. A set $A \subset X$ is said to be $\delta$-open [20] if it is the union of regular open sets of $X$. The complement of a regular open (resp. $\delta$-open) set is called regular closed (resp. $\delta$-closed). The intersection of all $\delta$-closed sets

2010 Mathematics Subject Classification. 54D10.

Key words and phrases. Ideal topological spaces, $\mathcal{I}$-open sets, almost $\mathcal{I}$-continuous functions. The authors are grateful to the refereee for his/her comments.

Communicated by: Takashi Noiri

${ }^{\dagger}$ Corresponding author. 